\title{
Long Non-Coding RNA DUXAP8 Facilitates Cell Viability, Migration, and Glycolysis in Non-Small-Cell Lung Cancer via Regulating HK2 and LDHA by Inhibition of miR-409-3p
}

This article was published in the following Dove Press journal: OncoTargets and Therapy

\section{Dianhe Yin ${ }^{1, *}$ \\ Li Hua ${ }^{2, *}$ \\ Jiao Wang \\ Yuru Liu (D) \\ Xiaoyan $\mathrm{Li}^{1}$}

'Department of General Practice, Huaihe Hospital of Henan University, Kaifeng 475000, Henan, People's Republic of China; ${ }^{2}$ Department of Respiratory and Critical Care Medicine, Peking University International Hospital, Beijing, People's Republic of China

*These authors contributed equally to this work
Correspondence: Yuru Liu

Department of General Practice, Huaihe Hospital of Henan University, Ximen Street II5, Longting District, Kaifeng 475000, Henan, People's Republic of China

Tel +86-0378-23906550

Email hfhghII8@I63.com
Purpose: Long non-coding RNAs (lncRNAs) were confirmed to play important roles in human cancers. In this study, we explored the functional role of IncRNA double homeobox A pseudogene 8 (DUXAP8) in non-small-cell lung cancer (NSCLC).

Methods: Real-time quantitative PCR (RT-qPCR) was used to detect DUXAP8 and microRNA-409-3p (miR-409-3p) expression. CCK-8, cell colony formation assay, and Transwell migration assay were performed to measure cell growth and migration, respectively. The expression of the relative proteins was detected by Western blot. Cell glycolysis was determined by glucose uptake, adenosine triphosphate (ATP) concentration, lactate generation, extracellular acidification rate and oxygen consumption rate assays. Bioinformatics analysis and dual-luciferase reporter assay were used to measure the interaction among DUXAP8, miR-409$3 p$, hexokinase 2 (HK2) and lactate dehydrogenase A (LDHA). In vivo, subcutaneous tumor formation assay was performed in the nude mice.

Results: DUXAP8 was highly expressed in NSCLC, while miR-409-3p was downregulated. High expression of DUXAP8 was positively related to the grade division and negatively associated with the 5-year survival rate of NSCLC patients. Downregulated DUXAP8 significantly suppressed cell growth, metastasis and glycolysis. Besides, DUXAP8 sponged miR-409-3p to promote HK2 and LDHA expression. DUXAP8 promoted cell viability, migration and glycolysis by regulating miR-409-3p/HK2/LDHA axis. Moreover, DUXAP8 downregulation markedly inhibited tumor growth in vivo.

Conclusion: Our findings demonstrated that DUXAP8 served as an oncogene in the progression of NSCLC.

Keywords: non-small-cell lung cancer, DUXAP8, miR-409-3p, HK2, LDHA

\section{Introduction}

Non-small-cell lung cancer (NSCLC), one of the leading causes related to cancer deaths, is the most common type of lung cancer accounting for about $80 \%{ }^{1}$ Unfortunately, approximately $80 \%$ NSCLC patients were diagnosed at the advanced stage. $^{2}$ Despite the molecular targeting therapy, chemotherapy and surgery had achieved advanced development, the 5-year survival rate of NSCLC patients is extremely poor, even less than $15 \% .^{3,4}$ NSCLC is a particularly complex tumor that is involved in the genetic dysregulation of tumor suppressor genes and protooncogenes, as well as multiple preneoplastic pathways. ${ }^{5}$ However, the biomarkers and molecular 
mechanisms for NSCLC early diagnosis, treatment, and prognosis remain largely lack and unclear.

Long non-coding RNAs (LncRNAs), with approximately 200 nucleotides, are a group of transcribed RNA sequences that do not possess the ability to encode proteins. ${ }^{6,7}$ LncRNAs played crucial regulatory roles in maintaining genome structure, gene transcription and translation, as well as epigenetic regulation. ${ }^{8,9}$ Accumulating evidence demonstrated the essential biological functions of lncRNAs in the tumorigenesis of multiple malignancies. ${ }^{10}$ The derived lncRNA double homeobox A pseudogene 8 (DUXAP8) with a length of $2107 \mathrm{bp}$ is located on chromosome 22q11.1. DUXAP8 was confirmed to facilitate the proliferation and metastasis of renal cell carcinoma cells, ${ }^{11}$ bladder cancer cells, ${ }^{12}$ and hepatocellular carcinoma cells. ${ }^{13}$ However, the effects and the underlying molecular mechanisms of DUXAP8 in NSCLC are still largely unknown.

MicroRNAs (miRNAs) are a series of non-coding, single-stranded RNAs with the length of 19 25 nucleotides that can lead to RNA degradation or translation inhibition of target mRNA through pairing with the 3 'untranslated region (3'UTR) of the target gene. ${ }^{14,15}$ MiRNAs were involved in the pathogenesis of varieties of human cancers, which could target mRNAs to regulate the tumorigenesis and the crucial biological processes of tumors, including cell growth, differentiation, metastasis, as well as cell apoptosis. ${ }^{16,17}$ Accumulating evidence revealed that dysregulation of miRNAs was closely correlated with the development of tumors. ${ }^{18}$ Several researches reported that microRNA-409$3 p$ (miR-409-3p) was aberrantly expressed in many malignant cancers, such as colon cancer, ${ }^{19}$ breast cancer, ${ }^{20}$ as well as NSCLC. ${ }^{21}$ Nevertheless, the functional effects of miR409-3p on NSCLC are not fully elucidated.

Aerobic glycolysis (also known as the Warburg effect), accompanying with the upregulated levels of lactate production and glucose uptake even in the condition with abundant oxygen, is regarded as a common and essential feature of cancer cells that can facilitate tumor growth. ${ }^{22,23}$ Thus, increasing researchers focused on the ectopic energy metabolism of cancer cells, which might be the novel potential pathway for cancer treatment. Hexokinase 2 (HK2) and lactate dehydrogenase A (LDHA) are essential drivers in the processes of glycolysis. HK2 is an enzyme participating in the initial and rate-limiting step of glycolysis that can convert glucose to glucose-6-phosphate. LDHA participates in the process through converting pyruvate into lactate, which is the final step of glycolysis. ${ }^{24,25}$ Increasing evidence suggested that HK2 and LDHA played promotion roles in human cancers. ${ }^{26,27}$ Thus, it is necessary to explore the functional effects of $\mathrm{HK} 2$ and LDHA on NSCLC progression.

In this research, the expression of DUXAP8 was determined in NSCLC. Functionally, the effects of DUXAP8 on the progression of NSCLC were explored both in vivo and in vitro. Mechanistically, the potential regulatory mechanism of DUXAP8 in the development of NSCLC was investigated.

\section{Patients and Methods Patients and Tissue Samples}

66 pairs of NSCLC tissues and the adjacent normal tissues were collected from 66 NSCLC patients, who were pathologically diagnosed as NSCLC and underwent surgery at Huaihe Hospital of Henan University. NSCLC patients did not treat with any preoperative anti-tumor methods. All participants provided the written informed consents. This study was approved by the Human Research Ethics Committee of Huaihe Hospital of Henan University.

\section{Cell Lines and Culture}

The normal human lung epithelial cells BEAS-2B and NSCLC cell lines (H1299, A549, H460 and PC-9) were used in this study. BEAS-2B, H1299, A549 and H460 cells were purchased from American Type Culture Collection (Manassas, VA, USA), and PC-9 cells were bought from Shanghai Zeye Biological Technology Co. Ltd. (Shanghai, China). BEAS-2B and A549 cells were maintained in Dulbecco's modified Eagle's medium (DMEM, Gibco, Carlsbad, CA, USA) and H1299, H460 and PC-9 cells were incubated with Roswell Park Memorial Institute 1640 (RPMI-1640) medium (Gibco), while DMEM and RPMI1640 were supplemented with streptomycin/penicillin (100 $\mathrm{U} / \mathrm{mL}$; Invitrogen, Carlsbad, CA, USA) and 10\% fetal bovine serum (FBS; Invitrogen). All the cells were cultured in a humidified chamber at $37^{\circ} \mathrm{C}$ with a condition of $5 \% \mathrm{CO}_{2}$.

\section{Cell Transfection}

Small interfering RNAs (siRNAs) targeting DUXAP8 (siDUXAP8 \#1: 5'- AAGATAAAGGTGGTTTCCACAAGA A-3'; si-DUXAP8 \#2: 5'-GGAACTTCCCAAACCTCCA TGATTT-3'; si-DUXAP8 \#3: 5'-CAGCATACTTCAAAT TCACAGCAAA-3') and the control si-NC (5'-UUCU CCGAACGUGUCACGUTT-3'), miR-409-3p mimic and the corresponding control group (NC mimic), DUXAP8 overexpression vector (DUXAP8) and the control vector 
(Vector), miR-409-3p inhibitor and the control (NC inhibitor) purchased from Genepharma (Shanghai, China) were transfected into A549 and H1299 cells using Lipofectamine 3000 Reagent (Invitrogen).

\section{Real-Time Quantitative Polymerase Chain Reaction (RT-qPCR)}

Total RNA was isolated from NSCLC tumorous tissues and the adjacent normal tissues, as well as NSCLC cells and BEAS-2B cells using Trizol reagent (Invitrogen). Briefly, total RNA was reverse-transcribed using SuperScript III RT (Invitrogen) under standard conditions, and then the amplified transcript level of the specific gene was determined by a real-time quantitative PCR system. The expression of miR409-3p and DUXAP8 was normalized by U6 and glyceraldehyde 3-phosphate dehydrogenase (GAPDH), respectively. The primer sequences of specific genes were as follows, DUXAP8: AGACGCCATGGAACAT (sense) and AAGCG GAGACCTGAGGAG (antisense), miR-409-3p: GGGGAA TGTTGCTCGGTGA (sense) and CAGTGCGTGTCGTGG AGT (antisense), U6: CTCGCTTCGGCAGCACA (sense) and AACGCTTCACGAATTTGCGT (antisense), GAPDH: AAGGCTGAGAATGGGAAAC (sense) and TTCAGGGA CTTGTCATACTTC (antisense). The relative expression of DUXAP8 and miR-409-3p was determined by the $2^{-\Delta \Delta C t}$ method.

\section{Cell Viability}

Cell viability was detected by Cell Counting Kit-8 (CCK-8) assay. $100 \mu \mathrm{L}$ cell suspension containing $2 \times 10^{3}$ cells was plated into the 96-well plates and then incubated for 24 h. Then, cells were cultured with $10 \mu \mathrm{L}$ CCK-8 solution (Dojindo, Tokyo, Japan). Finally, the absorbance was determined at $450 \mathrm{~nm}$ after transfection for $24 \mathrm{~h}, 48 \mathrm{~h}$, and $72 \mathrm{~h}$.

\section{Colony Formation Assay}

The selected A549 and H1299 cells with the density of 0.5 $\times 10^{3}$ cells per well were seeded into the 6 -well plates after transfection for $48 \mathrm{~h}$. Then, cells were incubated for 2 weeks. Subsequently, cells were washed using phosphatebuffered saline, fixed with methanol, stained with $0.1 \%$ crystal violet, and counted by a microscope (Bio-Rad Laboratories Inc., Hercules, CA, USA).

\section{Cell Migration}

The number of the migrated NSCLC cells was measured by transwell migration assay. After transfection, $3 \times 10^{4}$ cells maintained in serum-free medium were added into the higher chamber, while the lower chamber was supplemented with $500 \mu \mathrm{L}$ medium. After $48 \mathrm{~h}$ incubation, the not migrated cells were removed, while the migrated NSCLC cells on the lower chamber were fixed with $4 \%$ paraformaldehyde. Then, the migrated cells were stained with crystal violet, followed by counting in 5 randomly selected fields.

\section{Western Blot}

The involved protein expression was examined by Western blot analysis. Total protein was extracted from the transfected A549 and H1299 cells, as well as the mice tumor tissues by RIPA lysis buffer (Beyotime, Shanghai, China). $30 \mu \mathrm{g}$ total protein was separated by $10 \%$ sodium dodecyl sulfatepolyacrylamide gel electrophoresis. Then, the proteins were transferred onto a polyvinylidene difluoride (PVDF) membrane (Thermo Fisher Scientific, Waltham, MA, USA). Subsequently, the membrane was incubated with 5\% skim milk (dissolved in TBST buffer) for $1 \mathrm{~h}$ at room temperature. The proteins were incubated with the primary antibodies against c-myc (1:1000, 18,583, Cell Signaling Technology (CST), Boston, MA, USA), Cyclin D1 (1:200, ab16663, Abcam, Cambridge, UK), matrix metalloprotein 9 (MMP9, 1:1000, 13,667, CST), E-cadherin (E-cad, 1:1000; 14,472, CST), HK2 (1:1000, ab104836, Abcam), LDHA (1:2000, ab101562, Abcam) and GAPDH (1:1000, ab8245, Abcam) overnight at $4{ }^{\circ} \mathrm{C}$, followed by incubating with the secondary antibodies including goat anti-mouse IgG H\&L (1:2000, ab205719, Abcam) or goat anti-rabbit IgG H\&L (1:2000, ab6721, Abcam) for $2 \mathrm{~h}$. Finally, the signals of proteins were visualized by an electrochemiluminescent system (PerkinElmer Life Science, Waltham, MA, USA).

\section{Determination of Glucose Uptake, Lactate and ATP}

The levels of lactate production, glucose uptake, and ATP production were detected by Lactate Assay Kit II, Glucose Uptake Colorimetric Assay Kit, and ATP Colorimetric Assay Kit (BioVision, Milpitas, CA, USA) according to the manufacturer's instructions, respectively. For the examination of glucose uptake, the transfected cells with the density of $1 \times 10^{4}$ cells per well were seeded into the 96-well plate, followed by culturing in $100 \mathrm{~mL}$ KrebsRinger-Phosphate-HEPES (KRPH) buffer supplemented with $2 \%$ albumin from bovine serum for $40 \mathrm{~min}$. Then, $10 \mathrm{mM}$ 2-deoxyglucose (2-DG) was added and the cells 
were cultured for $20 \mathrm{~min}$. For the measurement of lactate and ATP production, $1 \times 10^{4}$ cells were suspended in the buffer from the corresponding assay kit. Then, the samples were centrifuged at $4^{\circ} \mathrm{C}$ for analyzing the soluble fraction.

\section{Extracellular Acidification (ECAR) and Oxygen Consumption Rate Assays (OCR)}

In the present study, we used the Seahorse Extracellular Flux Analyzer XF96 (Seahorse Bioscience, North Billerica, MA, USA) to calculate ECAR and OCR in NSCLC cells. After 48 $\mathrm{h}$ transfection, cells $\left(2 \times 10^{4}\right.$ per well $)$ were plated into the XF96-well plate and cultured overnight. For ECAR determination, glucose, oligomycin (oxidative phosphorylation inhibitor), and 2-DG (glycolytic inhibitor) were sequentially added into the NSCLC cells in culture. For OCR determination, cells were sequentially cultured with oligomycin, the reversible inhibitor of oxidative phosphorylation FCCP, and Rotenone/antimycin A. Data were analyzed by Seahorse XF96 Wave software. ECAR detection was noted as $\mathrm{mpH} / \mathrm{min}$, and OCR was represented as $\mathrm{pmol} / \mathrm{min}$.

\section{Dual-Luciferase Reporter Assay}

HEK293T cells were seeded into the 6-well plates and cultured for $24 \mathrm{~h}$. The wild-type sequences of DUXAP8 (WT-DUXAP8), HK2 (WT-HK2 3'UTR) and LDHA (WTLDHA 3'UTR) were constructed and inserted into pGL3 promoter vector (Invitrogen). The primer sequences for cloning DUXAP8 or the 3'-UTR of HK2 and LDHA were as below: 5'-GGTGGGTTCCATGTGATGGT-3' (Forward) and 5'-GTCTTCTGAGAAACATTGTGCCA -3' (Reverse) for DUXAP8; 5'-TGGTGTTAGGAAAG TCGCCC-3' (Forward) and 5'-AGGAAGGGCCTACTC ACAGT-3' (Reverse) for HK2; 5'-TAGTCTGATTTCCGC CCACC-3' (Forward) and 5'-ACCGCTTCCAATAACA CGGT-3' (Reverse) for LDHA. The potential DUXAP8, HK2 and LDHA binding sites for miR-409-3p were mutated by Quick-change site-directed mutagenesis kit (Agilent Technologies, Santa Clara, CA, USA), named as MUT-DUXAP8, MUT-HK2 3'UTR and MUT-LDHA 3'UTR reporter plasmids. Then, HEK293T cells were cotransfected with $400 \mathrm{ng}$ of these constructed plasmids (WT-DUXAP8, WT-HK2 3'UTR, WT-LDHA 3'UTR or MUT-DUXAP8, MUT-HK2 3'UTR, MUT-LDHA 3'U $\mathrm{TR}$ ), $50 \mathrm{ng}$ of renilla luciferase reporter plasmid (pRL$\mathrm{TK}$ ) and $50 \mathrm{nM}$ of miR-409-3p mimic or NC mimic using Lipofectamine 3000 (Invitrogen). After $48 \mathrm{~h}$ transfection, the relative luciferase activity was measured by a dualluciferase reporter assay system (Promega, Madison, WI, USA). Renilla luciferase activity was used as normalization.

\section{Xenograft Experiment}

The 4-6 weeks old BALB/c nude mice bought from Beijing Laboratory Animal Center (Beijing, China) were used in this study. A549 cells were stably transfected with short hairpin RNA against DUXAP8 (sh-DUXAP8) or shNC (Genepharma, Shanghai, China). Approximately $1 \times$ $10^{6}$ sh-DUXAP8/sh-NC-infected A549 cells were subcutaneously inoculated into the flanks of the nude mice (5 per group). Tumor volume was detected every 3 days from 6 day using a caliper. After 30 days, the mice were euthanized and tumor weight was assessed. All experiments were performed in line with the Guide for the Care and Use of Laboratory Animals and approved by the Huaihe Hospital of Henan University Experimental Animal Ethics Committee.

\section{Statistical Analysis}

All data were represented as the mean \pm the standard deviations (SD) and analyzed by SPSS 18.0 software. Comparisons between groups were calculated using Student's $t$-test or Oneway ANOVA. Pearson correlation analysis was used to analyze the relationship between miR-409-3p and DUXAP8. Kaplan-Meier was used to measure the overall survival. $P<$ 0.05 was regarded as statistically significant.

\section{Results DUXAP8 Was Upregulated and Associated with Low Overall Survival in NSCLC Patients}

We detected the expression of DUXAP8 in 66 tumorous tissues and the paired adjacent normal tissues from NSCLC patients using RT-qPCR. A significant increase of DUXAP8 was observed in tumor tissues compared with the normal tissues (Figure 1A). The data showed that DUXAP8 expression was markedly upregulated in 51 NSCLC tissues (Figure 1B). The data in Figure 1C suggested that DUXAP8 was significantly higher in the advanced Tumor Node Metastasis stage (Stage III + IV) relative to the low Stage I + II. We also determined the association between DUXAP8 and lymph node metastasis and found that high expression of DUXAP8 was positively correlated with lymph node metastasis in NSCLC patients (Figure 1D). Besides, the overall 
survival of NSCLC patients with high DUXAP8 expression was shorter than the low DUXAP8 expression group (Figure 1E). These results demonstrated that DUXAP8 served as an oncogenic role in NSCLC and higher expression of DUXAP8 was correlated with shorter overall survival.

\section{DUXAP8 Knockdown Inhibited Cell Viability and Migration of NSCLC Cells}

Next, we measured the expression of DUXAP8 in NSCLC cells and BEAS-2B cells. RT-qPCR showed that DUXAP8 expression was higher in NSCLC cells (Figure 2A). To determine the biological functions of DUXAP8 in NSCLC, siRNAs against DUXAP8 were transfected into A549 and H1299 cells, which had the highest expression of DUXAP8. The data suggested that siRNAs against DUXAP8 significantly decreased the expression of DUXAP8 in both A549 and H1299 cells (Figure 2B). Next, si-DUXAP8 \#1 was selected to perform the following experiments, which exerted the best knockdown efficiency in NSCLC cells. CCK-8 assay indicated that cell viability in A549 and H1299 cells transfected with si-DUXAP8 at $48 \mathrm{~h}$ and 72 $\mathrm{h}$ was markedly inhibited in comparison to the control group (Figure 2C and D). Consistently, the number of colonies was significantly attenuated by DUXAP8 knockdown
(Figure 2E). Moreover, transwell migration assay demonstrated that DUXAP8 knockdown could decrease the number of migrated NSCLC cells (Figure 2F). Subsequently, we detected the expression of cell viability-related proteins c-myc and Cyclin D1, as well as metastasis-related proteins MMP9 and E-cad. As described in Figure 2G and H, DUXAP8 knockdown significantly suppressed the expression of c-myc, Cyclin D1 and MMP9, and enhanced E-cad expression in both A549 and H1299 cells. All these results indicated that DUXAP8 downregulation inhibited cell growth and metastasis in NSCLC cells.

\section{DUXAP8 Downregulation Dampened Glycolysis in NSCLC Cells}

Glycolysis is related to multiple reactions that each glucose molecule can be converted to pyruvate to produce 2 adenosine triphosphate (ATP). The consumption of glucose in cancer cells can produce lactate, which is the final metabolism product of glycolysis, even in the environment with oxygen. Thus, we investigated whether DUXAP8 affected the glycolysis of NSCLC cells. DUXAP8 knockdown decreased glucose uptake, lactate production and ATP generation (Figure 3A-C). Additionally, the indicators of glycolysis, including ECAR and OCR, were
A

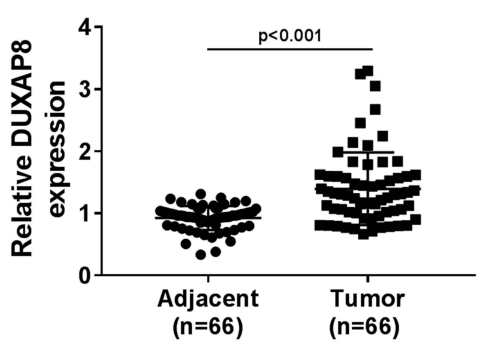

D

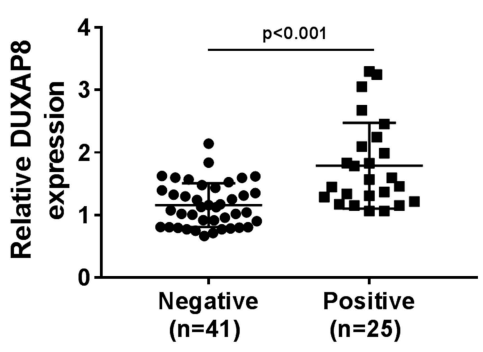

B

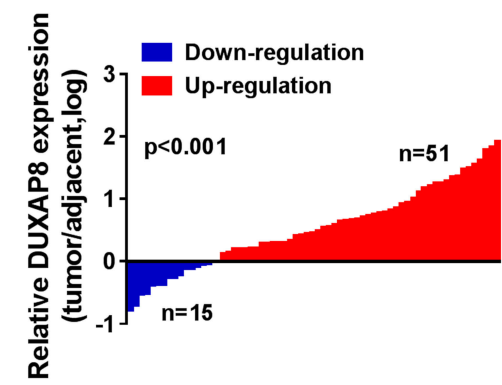

$E$

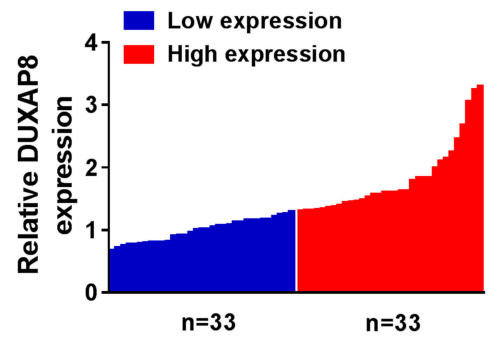

C
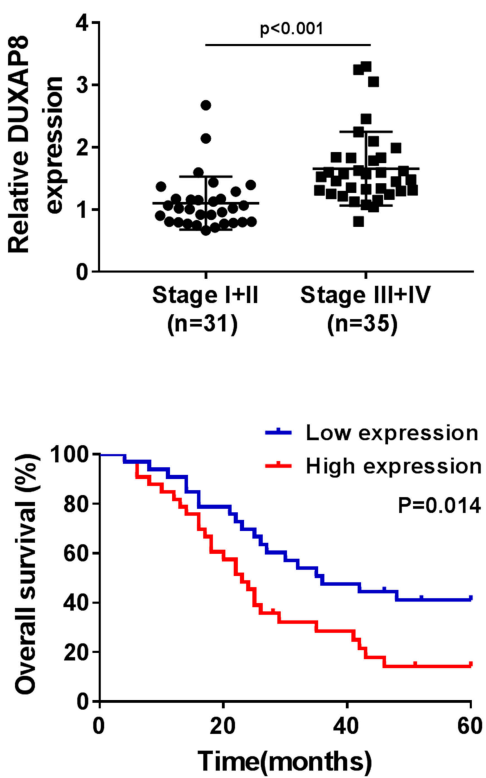

Figure I DUXAP8 was upregulated and associated with the low overall survival rate in NSCLC patients. (A) The expression of DUXAP8 in NSCLC tissues ( $\mathrm{n}=66$ ) and the adjacent normal tissues $(n=66)$ was detected by RT-qPCR. (B) RT-qPCR analysis was used to measure DUXAP8 expression in 66 pairs of NSCLC tissues and the corresponding non-tumor lung tissues. (C) DUXAP8 expression was examined in tumor tissues isolated from various TNM stage (I+II ( $n=3 \mid$ ), III+IV ( $=35)$ ) NSCLC patients by RT-qPCR. (D) DUXAP8 expression in lymph node metastatic $(n=4 I)$ and nonmetastatic $(n=25)$ NSCLC tissues was assessed by RT-qPCR. (E) Kaplan-Meier survival curves and Log rank tests were used to explore the relationship between DUXAP8 expression and the 5-year overall survival rate of NSCLC patients; low DUXAP8 expression group $(n=33)$ and high DUXAP8 expression group $(n=33)$. 
A

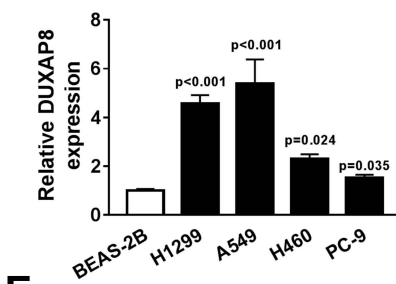

E

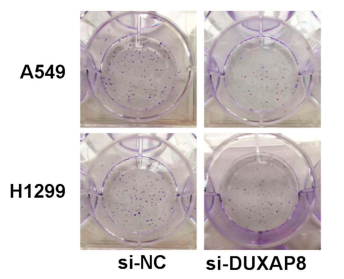

G

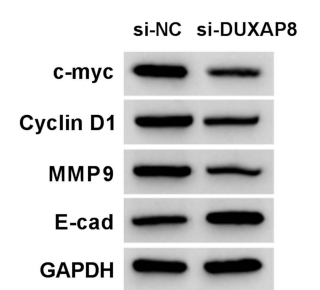

B
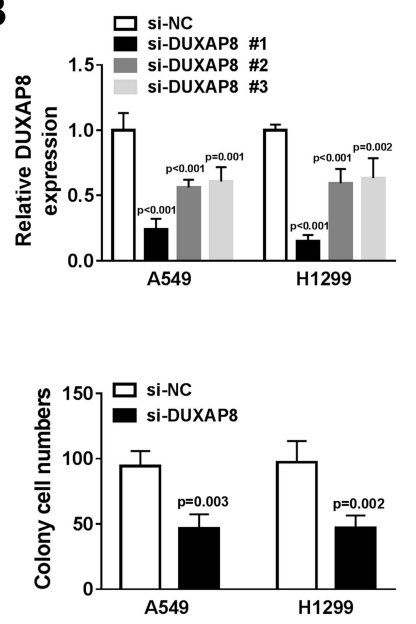

A549

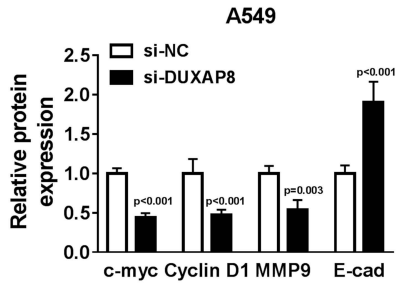

C

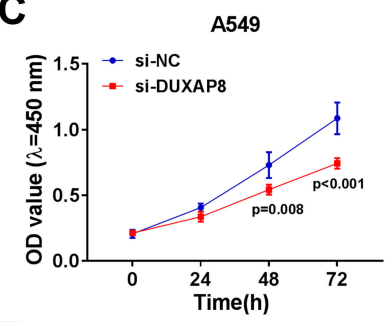

F

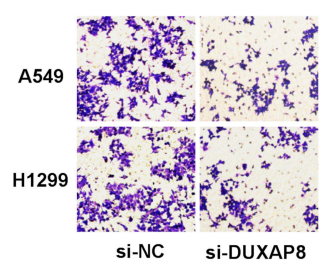

H

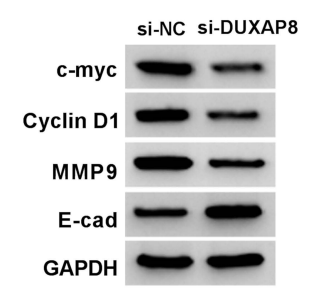

D
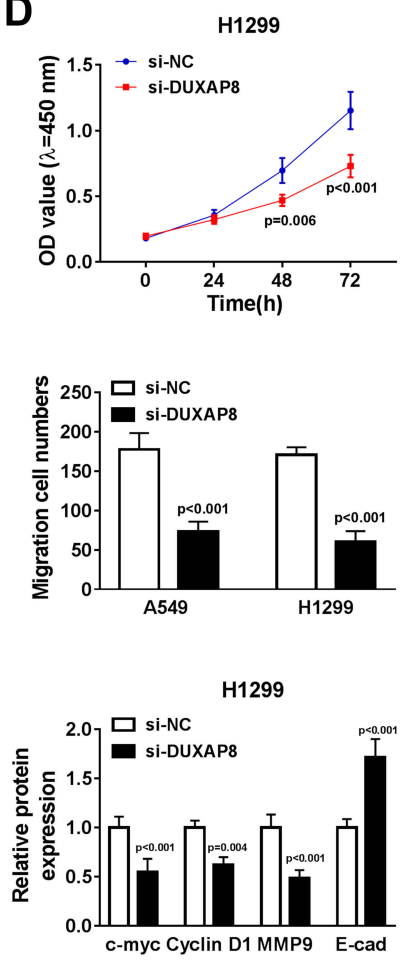

Figure 2 DUXAP8 knockdown inhibited the viability and migration of NSCLC cells. (A) DUXAP8 expression in BEAS-2B, HI299, A549, H460 and PC-9 cells was detected by RT-qPCR $(n=3)$. (B) RT-qPCR was used to measure the expression of DUXAP8 in A549 and HI299 cells transfected with siRNAs against DUXAP8 or si-NC ( $\mathrm{n}=3$ ). (C and D) CCK-8 assay was used to determine the viability of NSCLC cells transfected with si-DUXAP8 or si-NC ( $n=3$ ). (E) The number of colonies of NSCLC cells transfected with si-DUXAP8 or si-NC was detected by cell colony formation assay $(n=3)$. (F) The migratory ability of NSCLC cells transfected with si-DUXAP8 or si-NC was examined by transwell migration assay $(n=3)$. ( $(\mathbf{G}$ and $\mathbf{H})$ The protein expression of c-myc, Cyclin DI, MMP9 and E-cad in NSCLC cells transfected with si-DUXAP8 or si-NC was detected by Western blot $(n=3)$.

calculated. ECAR can reflect overall glycolytic flux, while OCR can reflect mitochondrial oxidative respiration. As shown in Figure 3D and E, downregulation of DUXAP8 could significantly enhance OCR in both A549 and H1299 cells. DUXAP8 knockdown markedly suppressed ECAR in NSCLC cells (Figure 3F and G). We further measured the glycolysis-related proteins including HK2 and LDHA. The data suggested that DUXAP8 knockdown significantly reduced the expression of HK2 and LDHA in both A549 and H1299 cells (Figure 3H and I). These results collectively demonstrated that DUXAP8 knockdown inhibited the glycolysis in NSCLC cells.

\section{DUXAP8 Targeted MiR-409-3p to}

\section{Regulate HK2 and LDHA Expression}

To explore whether DUXAP8 functioned as a competing endogenous RNA, we searched DUXAP8 binding miRNAs using StarBase v2.0, which was further used to predict the potential binding miRNAs of HK2 and LDHA. Venn diagram showed that 101 miRNAs harbored the binding sites of DUXAP8, 197 miRNAs harbored the binding sites of HK2, and 85 miRNAs harbored the binding site of LDHA (Figure 4A). We found that there were 11 miRNAs simultaneously contained the binding sits of DUXAP8, HK2 and LDHA. Among the 11 miRNAs, miR-409-3p was identified as a candidate miRNA. RT-qPCR showed that the expression of miR-409-3p was significantly decreased in NSCLC tissues and cells (Figure 4B and C). Besides, we discovered a negative relationship between DUXAP8 and miR-409-3p in NSCLC tissues (Figure 4D). As described in Figure 4E, miR-409-3p harbored the binding sites of DUXAP8, HK2 and LDHA. To verify the predicted relationships, dual-luciferase reporter assay was carried out and the results suggested that the luciferase activity of the wild-type (WT) of DUXAP8, HK2 3'UTR and LDHA 3'UTR reporter vectors, but not (MUT) vectors, was markedly decreased in HEK293T cells transfected with miR-409-3p mimic compared with NC mimic group (Figure 4F-H).

Moreover, RT-qPCR indicated a successful overexpression efficiency of DUXAP8 in both A549 and H1299 cells (Figure 4I). As shown in Figure 4J, the expression of miR409-3p was significantly increased by downregulating 


\section{A}

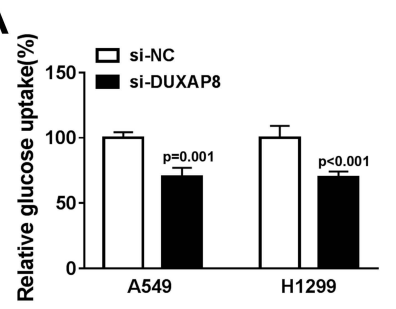

D

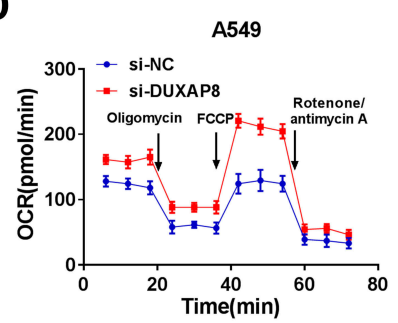

F

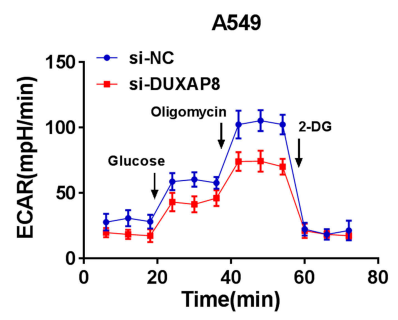

H

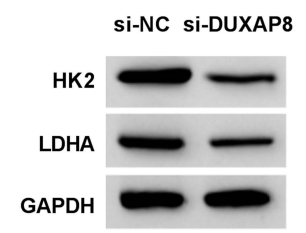

B
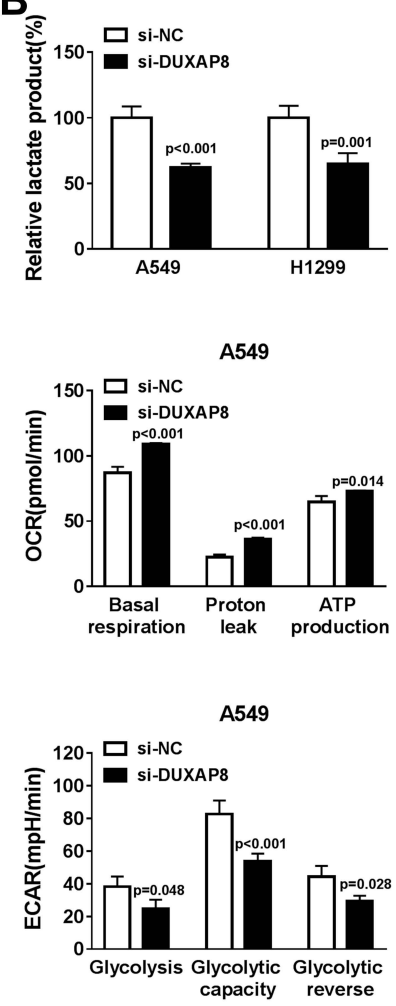

A549

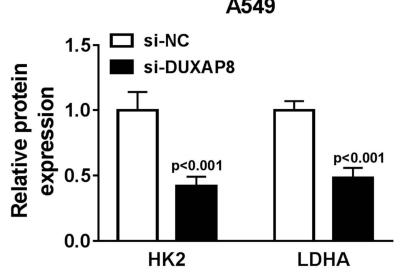

C

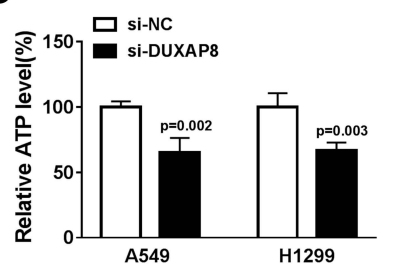

E

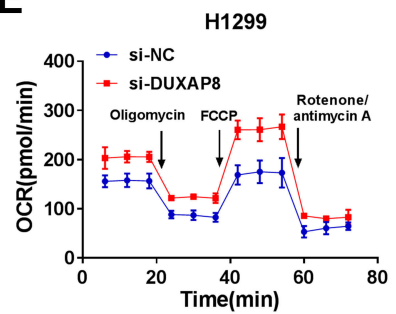

G

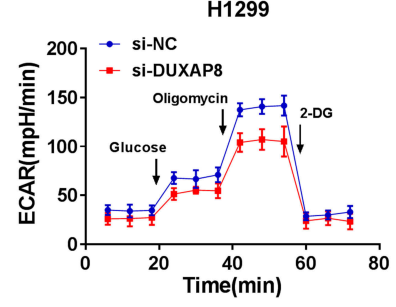

I

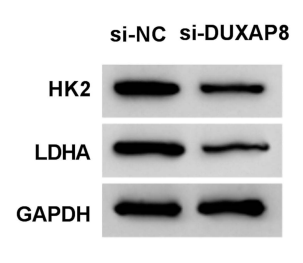

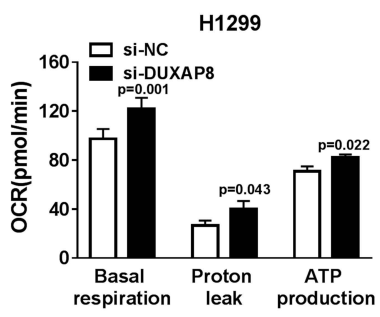

H1299

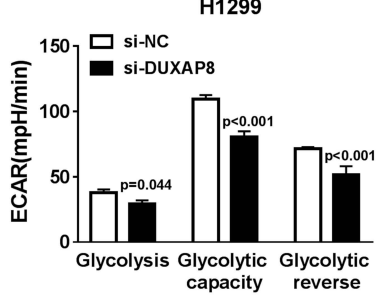

H1299

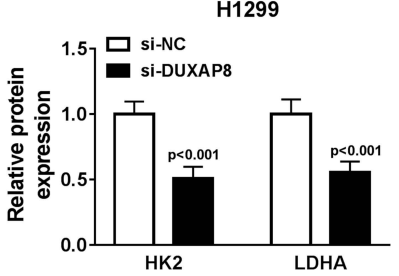

Figure 3 DUXAP8 downregulation dampened the glycolysis in NSCLC cells. (A-C) Glucose uptake (A), lactate release (B), and ATP production level (C) were measured in A549 and HI299 cells transfected with si-DUXAP8 or si-NC by Glucose Uptake Colorimetric Assay Kit, Lactate Assay Kit II, and ATP Colorimetric Assay Kit, respectively $(n=3)$. ( $\mathbf{D}-\mathbf{G})$ The quantification of OCR ( $\mathbf{D}$ and $\mathbf{E})$ and ECAR ( $\mathbf{F}$ and $\mathbf{G})$ in NSCLC cells transfected with si-DUXAP8 or si-NC was measured by Seahorse Extracellular Flux Analyzer XF96 assays $(n=3)$. ( $(H$ and $\mathbf{I})$ The protein expression of HK2 and LDHA in NSCLC cells transfected with si-DUXAP8 or si-NC was examined by Western blot analysis $(n=3)$.

DUXAP8 and decreased by overexpressing DUXAP8 in NSCLC cells. Furthermore, miR-409-3p expression was upregulated in NSCLC cells transfected with miR-409-3p mimic, while the expression of miR-409-3p was downregulated in NSCLC cells transfected with miR-409-3p inhibitor (Figure 4K). We also detected the expression of HK2 and LDHA in A549 and H1299 cells transfected with miR-409-3p mimic, NC mimic, miR-409-3p inhibitor, or $\mathrm{NC}$ inhibitor, and the results suggested that the expression of HK2 and LDHA was markedly inhibited by overexpression of miR-409-3p, but enhanced by downregulation of miR-409-3p (Figure 4L). Taken together, we observed that DUXAP8 sponged miR-409-3p to regulate the expression of HK2 and LDHA.

\section{MiR-409-3p Inhibitor Reversed Downregulation of DUXAP8-Mediated Cell Viability and Migration}

To explore whether miR-409-3p was involved in DUXAP8-induced promotion effects on NSCLC cell viability and migration, we performed rescue experiments by co-transfecting si-DUXAP8 and miR-409-3p inhibitor into A549 and H1299 cells. As described in Figure 5A and B, downregulation of DUXAP8-impaired cell viability of NSCLC cells was reversed by miR-409-3p inhibitor. Besides, miR-409-3p downregulation rescued the reduction of the number of colonies and the migrated NSCLC cells transfected with si-DUXAP8 (Figure 5C and D). 
A

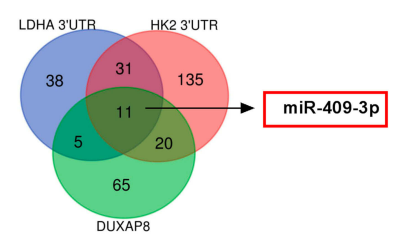

E

Binding Site chr22:16148160-16148166[-]

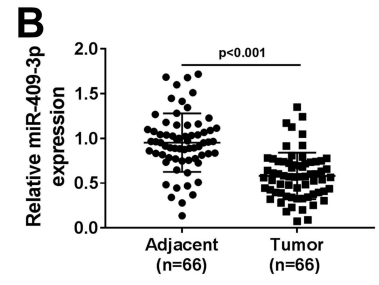

F

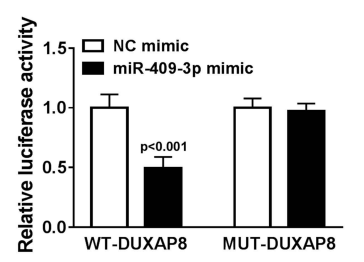

WT-HK2 3'UTR 5' AAAACCUCAGGGACAACAUUU 3'

miR-409-3p 3' UccCCAAGUGGCUCGUUGUAAG 5

MUT-HK2 3'UTR 5' AAAACCUCAGGGAGUUGUAAU 3'

Binding Site chr11:18429185-18429191[+]

WT-LDHA 3'UTR 5' GAACAUGCCUAGUCCAACAUUU 3'

miR-409-3p 3' UCCCCAAGUGGCUCG II II | ||

MUT-LDHA 3'UTR 5' GAACAUGCCUAGUCGUUGUAAU 3'

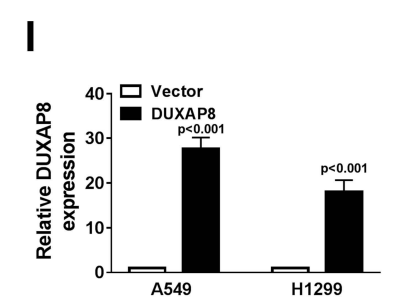

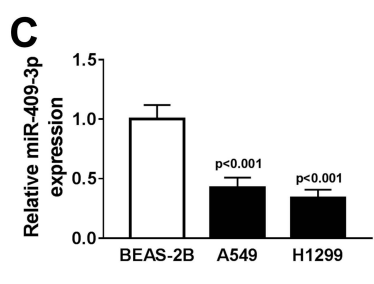

G

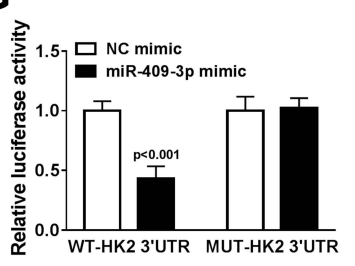

J
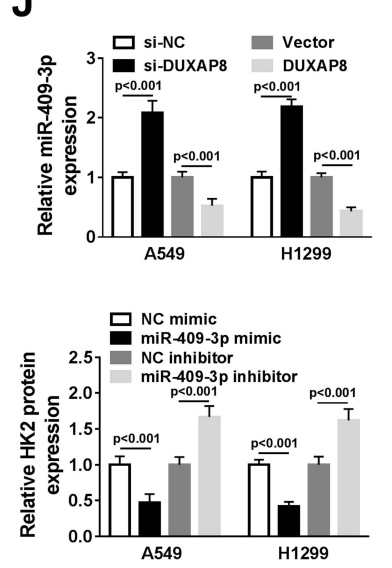

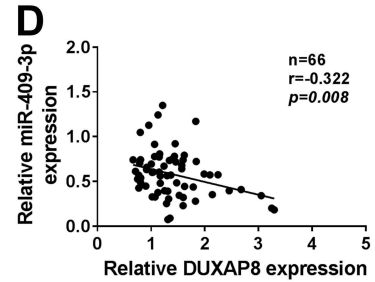

H
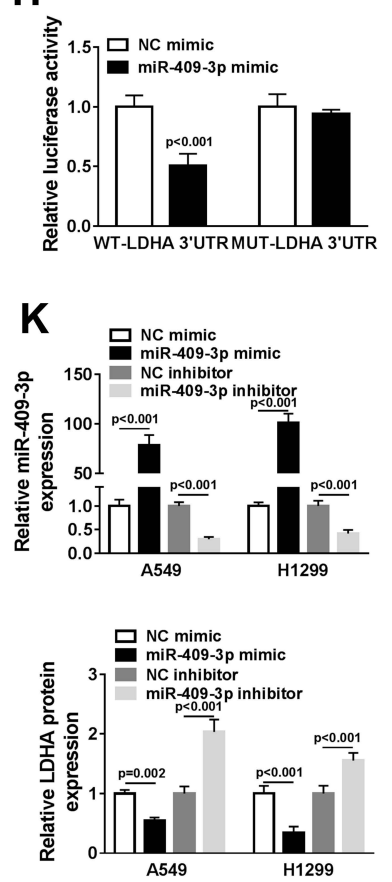

L
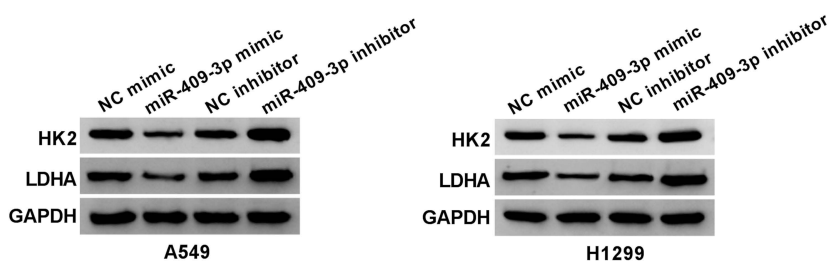

Figure 4 DUXAP8 targeted miR-409-3p to regulate HK2 and LDHA expression. (A) The overlapped binding miRNAs were analyzed by Venn diagram. (B) The expression of miR-409-3p in NSCLC tissues was detected by RT-qPCR $(n=3)$. (C) RT-qPCR was used to measure the expression of miR-409-3p in BEAS-2B, A549 and HI299 cells $(n=3)$. (D) The relationship between DUXAP8 and miR-409-3p in NSCLC tissues was analyzed by Kaplan-Meier analysis $(r=-0.322, P=0.008)(n=3)$. $(E)$ StarBase $v 2.0$ predicted that miR-409-3p contained the binding sites of DUXAP8, HK2 and LDHA. (F and $\mathbf{H})$ Dual-luciferase reporter assay was performed to determine the relative luciferase activity in 293T cells co-transfected with wild type (WT-) or mutant (MUT-) of DUXAP8, HK2 3'UTR or LDHA 3'UTR and miR-409-3p mimic or NC mimic ( $n=3$ ). (I) The expression of DUXAP8 in NSCLC cells transfected with DUXAP3 or Vector was detected by RT-qPCR $(n=3)$. (J) RT-qPCR was used to determine miR-409-3P expression in NSCLC cells transfected with si-NC, si-DUXAP8, Vector, or DUXAP8 $(n=3)$. (K) RT-qPCR was used to detect the expression of miR-409-3p in NSCLC cells transfected with NC mimic, miR-409-3p mimic, NC inhibitor, or miR-409-3p inhibitor ( $n=3)$. (L) The protein expression of HK2 and LDHA in NSCLC cells transfected with NC mimic, miR-409-3p mimic, NC inhibitor, or miR-409-3p inhibitor was measured by Western blot $(n=3)$.

Subsequently, the involvement of protein expression, including c-myc, Cyclin D1, MMP9 and E-cad, was detected by Western blot. DUXAP8 downregulation induced the decrease of c-myc, Cyclin D1 and MMP9 expression, as well as the increase of E-cad expression in NSCLC cells, which were reversed by downregulating miR-409-3p (Figure 5E and F).

\section{MiR-409-3p Inhibitor Reversed the Inhibitory Effect of DUXAP8 Downregulation on Glycolysis}

To confirm whether DUXAP8 might affect glycolysis via targeting miR-409-3p in NSCLC cells, we measured glucose uptake, lactate production and ATP generation. The data demonstrated that miR-409-p knockdown blocked the inhibitory effects of DUXAP8 knockdown on glucose uptake, lactate and ATP production in both A549 and H1299 cells (Figure 6A-C). Subsequently, we detected the ECAR and OCR in NSCLC cells transfected with si-NC, si-DUXAP8, siDUXAP8+NC inhibitor, or si-DUXAP8+miR-409-3p inhibitor. OCR was facilitated by DUXAP8 downregulation in NSCLC cells, while miR-409-3p inhibitor could significantly attenuate OCR in both A549 and H1299 cells (Figure 6D and $\mathrm{E}$ ). We also discovered that miR-409-3p inhibitor significantly reversed the reduction of ECAR induced by DUXAP8 knockdown in NSCLC cells (Figure 6F and G). Besides, Western blot analysis indicated that the suppressive effects of DUXAP8 knockdown on HK2 and LDHA expression were reversed by downregulating miR-409-3p in both A549 and H1299 cells (Figure 6H and I). Overall, these data suggested 

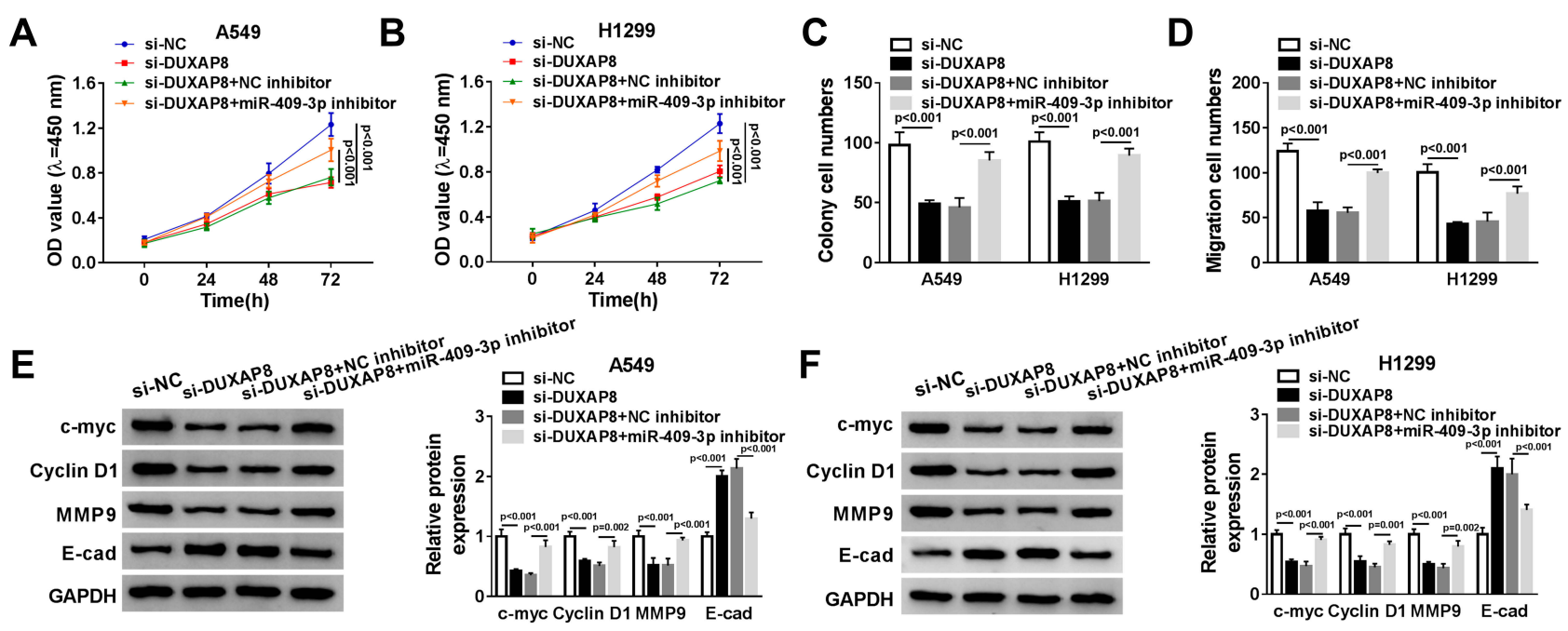

Figure 5 MiR-409-3p inhibitor reversed DUXAP8 downregulation-mediated cell viability and migration. A549 and HI 299 cells were transfected with si-NC, si-DUXAP8, siDUXAP8+NC inhibitor, or si-DUXAP8+miR-409-3p inhibitor. (A and B) Cell viability was detected by CCK-8 assay $(n=3)$. (C and $\mathbf{D})$ The number of colonies was assessed by cell colony formation assay $(n=3)$. (E and $\mathbf{F})$ The protein expression of c-myc, Cyclin DI, MMP9 and E-cad was determined by Western blot analysis ( $\mathrm{n}=3$ ).

that miR-409-3p inhibitor reversed downregulation of DUXAP8-mediated NSCLC cell glycolysis.

\section{DUXAP8 Knockdown Suppressed Tumor Growth in vivo}

To validate the effect of DUXAP8 on tumor growth in vivo, we constructed A549 cells stably transfected with shDUXAP8 or sh-NC. Then, A549 cells stably transfected with sh-DUXAP8 or sh-NC were inoculated into the nude mice. We found that tumor volume was markedly suppressed in the sh-DUXAP8 mice group compared with the control group (Figure 7A). Moreover, tumor weight was also lower in the sh-DUXAP8 group than that in the si-NC group (Figure 7B). RT-qPCR analysis showed that the expression of DUXAP8 was lower, while miR-409-3p expression was higher in tumor tissues from the mice of the sh-DUXAP8 group than that in the control group (Figure 7C). We further determined the protein expression of HK2 and LDHA and discovered that downregulation of DUXAP8 could significantly decrease HK2 and LDHA expression in the tumor tissues from the nude mice (Figure 7D). These in vivo findings confirmed that DUXAP8 was essential for promoting tumor growth in NSCLC.

\section{Discussion}

Growing evidence confirmed that lncRNAs with ectopic expression were commonly observed in various human cancers, which might be biomarkers for the prediction, diagnosis, or prognosis of cancers. ${ }^{28}$ NSCLC is the most common malignancy in the lung, and the underlying mechanisms of NSCLC progression remain largely unknown. Recently, lncRNA DUXAP8 was confirmed to serve as an essential role in the occurrence and development of various cancers. For instance, Huang and his colleagues indicated that DUXAP8 expression was dramatically increased in glioma tissues, which was closely associated with the prognosis of glioma patients and exhibited significant promotion effects on cell growth. ${ }^{29}$ Thus, we determined the expression and functional role of DUXAP8 in NSCLC. Consistent with the results in the research from Sun and his colleagues, ${ }^{30}$ we discovered that the expression of DUXAP8 in NSCLC tissues and cells was significantly increased. Moreover, DUXAP8 expression was correlated with the TNM stage and positively related to the lymph node metastasis in NSCLC patients. Then, we performed the functional experiments to investigate the biological functions of DUXAP8 in NSCLC. We found that downregulation of DUXAP8 exerted significant inhibition effects on cell growth and migration in both A549 and H1299 cells. Meanwhile, DUXAP8 knockdown markedly reduced the expression of c-myc, Cyclin D1 and MMP9, and upregulated E-cad expression in NSCLC cells. We demonstrated that DUXAP8 played a promotion role in the progression of NSCLC in vitro. Furthermore, DUXAP8 knockdown suppressed tumor growth in NSCLC in vivo.

Recently, multiple researchers reported that the ectopic metabolism in cancer cells with the upregulated glucose uptake and lactate production was proved to improve the biological processes of cancer. ${ }^{31}$ Several lncRNAs were 
A

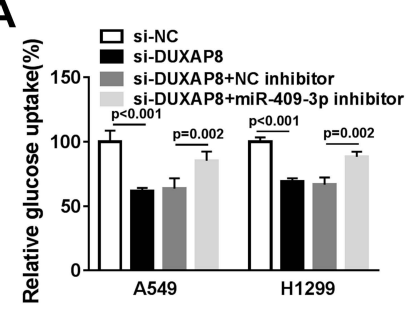

D
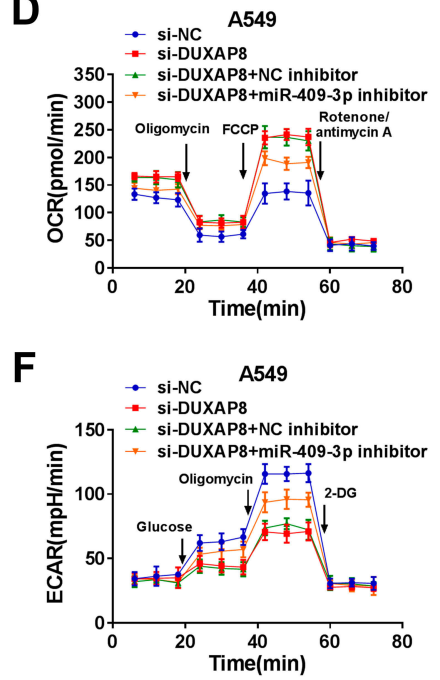

H

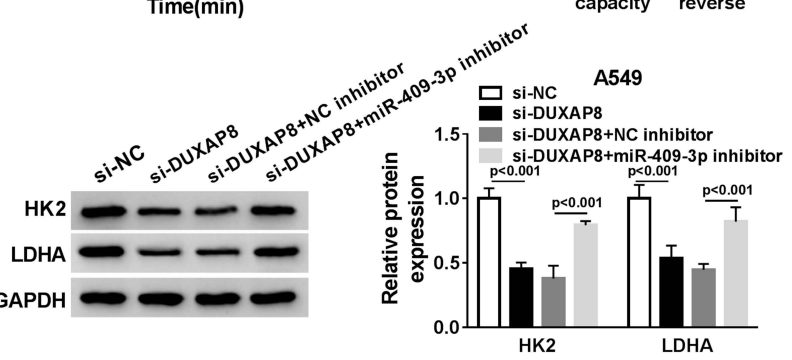

B
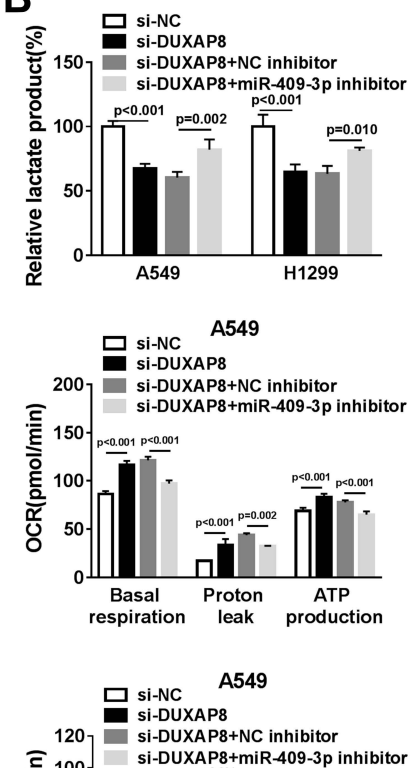

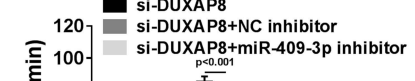

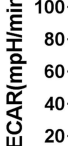

每

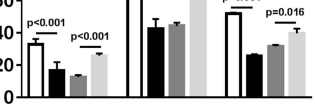

Glycolysis Glycolytic Glycolytic

\section{G}
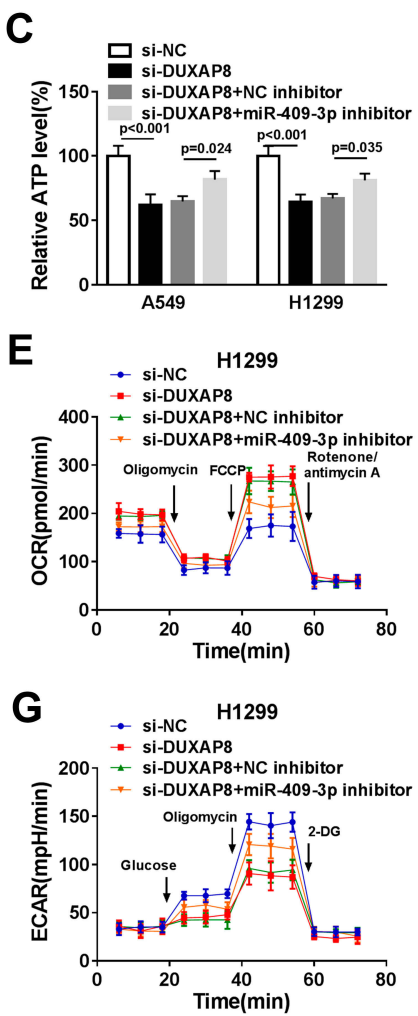

E
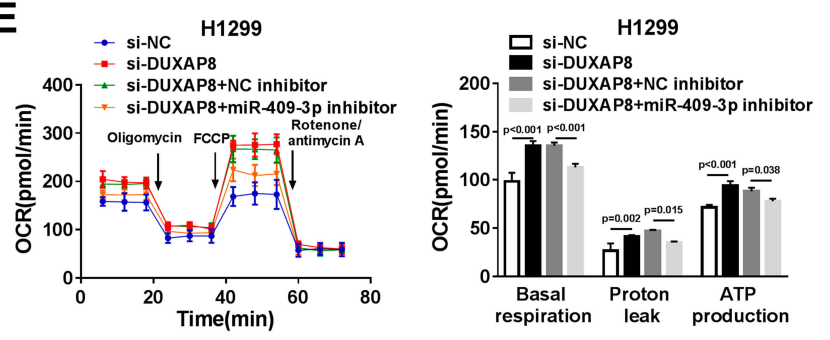

I
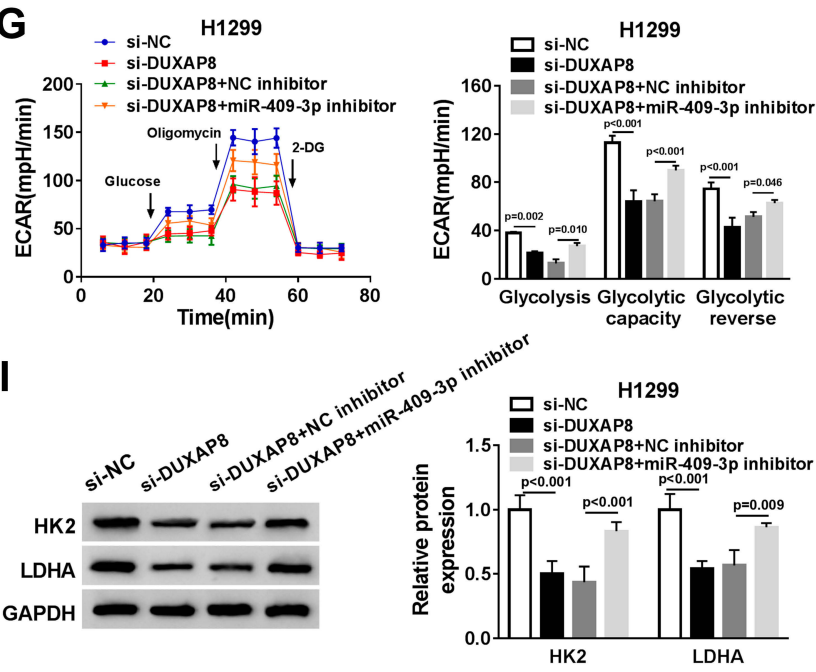

Figure 6 MiR-409-3p inhibitor reversed the inhibitory effect of DUXAP8 downregulation on the glycolysis. A549 and HI299 cells were transfected with si-NC, si-DUXAP8, si-DUXAP8+NC inhibitor, or si-DUXAP8+miR-409-3p inhibitor. (A-C) The relative glucose uptake (A), lactate production (B), and ATP production (C) in A549 and HI299 cells were measured by Glucose Uptake Colorimetric Assay Kit, Lactate Assay Kit II, and ATP Colorimetric Assay Kit, respectively ( $n=3)$. (D-G) The quantification of OCR (D and E) and ECAR (F and G) in A549 and HI299 cells was calculated by Seahorse Extracellular Flux Analyzer XF96 assays ( $\mathrm{n=3}$ ). (H and I) Western blot analysis was used to examine the expression of HK2 and LDHA in A549 and HI299 cells ( $n=3)$.

demonstrated to be intimately related to regulating aerobic glycolysis to facilitate cancer cell survival and growth. ${ }^{32}$ For example, PCGEM1 acted as a key regulator to reprogram energy metabolism through directly targeting c-myc in cancer. ${ }^{33}$ LINC01123 was highly expressed in NSCLC and facilitated cell growth, as well as aerobic glycolysis through targeting miR-199a-5p. ${ }^{34}$ In another study, IncRNA UCA1 exerted promotion effects on tumorigenesis through overexpressing the expression of HK2 in bladder cancer. ${ }^{35}$ Consistent with the previous research, our results indicated that DUXAP8 downregulation could inhibit uptake glucose, lactate release and ATP production in NSCLC cells. Furthermore, the key metabolic enzymes HK2 and LDHA played crucial roles in the processes of aerobic glycolysis, which participated in the progression of cancers. ${ }^{36,37}$ In this study, knockdown of DUXAP8 markedly repressed the expression of HK2 and LDHA in NSCLC cells.

Recently, multiple miRNAs, such as miR-181b, ${ }^{38}$ miR$98,{ }^{39}$ and miR-449a, ${ }^{40}$ were confirmed to suppress HK2 or LDHA expression. In our study, we discovered that miR-409$3 p$ harbored the binding sites of DUXAP8, HK2 and LDHA. Besides, DUXAP8 could directly sponge miR-409-3p to regulate HK2 and LDHA in NSCLC cells. Previous researches indicated that miR-409-3p functioned as a suppressor in the progression of lung cancer. ${ }^{41,42}$ Consistently, we observed that miR-409-3p was lowly expressed in NSCLC tissues and cells. Besides, miR-409-3p downregulation could reverse the effects of DUXAP8 knockdown on cell growth, migration and glycolysis, which further rescued by the reduction of HK2 and LDHA expression in NSCLC cells. 
A

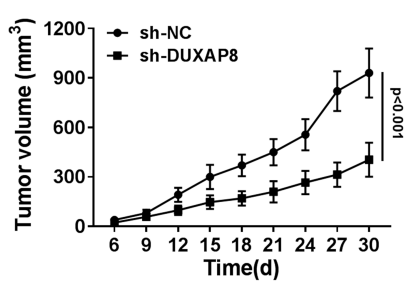

D

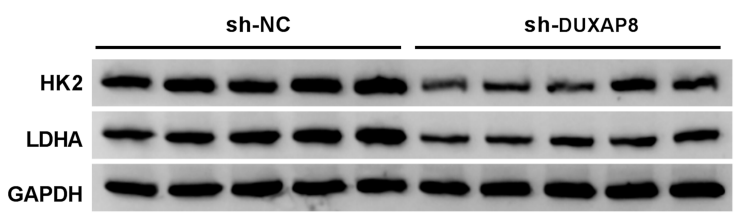

B

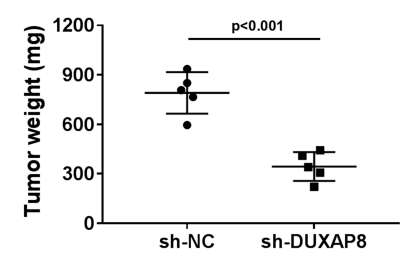

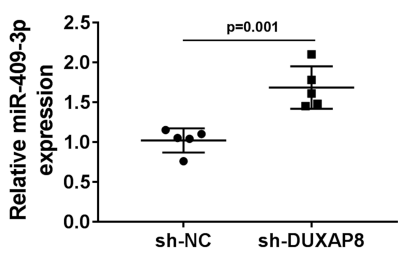

sh-NC sh-DUXAP8

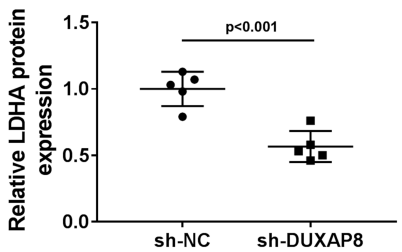

Figure 7 DUXAP8 knockdown suppressed tumor growth in vivo. (A and B) Tumor volume (A) and weight (B) of the nude mice injected with sh-NC or sh-DUXAP8 were measured $(n=5)$. (C) RT-qPCR was used to measure the expression of DUXAP8 and miR-409-3p in tumor tissues $(n=3)$. (D) The protein expression of HK2 and LDHA in tumor tissues was detected by Western blot analysis $(n=3)$.

\section{Conclusion}

In conclusion, DUXAP8 was confirmed to be highly expressed in NSCLC tissues and cells, which was associated with the grade division and prognosis of NSCLC patients. Functionally, DUXAP8 promoted NSCLC cell growth, metastasis and glycolysis. Mechanistically, we found that DUXAP8 was involved in NSCLC progression by inhibiting miR-409-3p to regulate HK2 and LDHA expression. Our study provided a potential target for the treatment and prognosis of NSCLC, which could help to further understand the molecular mechanisms and progression of NSCLC.

\section{Highlights}

1. DUXAP8 was highly expressed in non-small-cell lung cancer tissues and cells.

2. DUXAP8 enhanced non-small-cell lung cancer cell viability, migration and glycolysis.

3. DUXAP8 increased HK2 and LDHA expression by targeting miR-409-3p.

4. DUXAP8 promoted non-small-cell lung cancer cell viability, migration and glycolysis via targeting miR409-3p to regulate HK2 and LDHA expression.

\section{Data Sharing Statement}

The analyzed data sets generated during the present study are available from the corresponding author on reasonable request.

\section{Ethics Approval and Consent to Participate}

The present study was approved by the ethical review committee of Huaihe Hospital of Henan University.

\section{Author Contributions}

All authors contributed to data analysis, drafting or revising the article, gave final approval of the version to be published, and agree to be accountable for all aspects of the work.

\section{Funding}

No funding was received.

\section{Disclosure}

The authors declare that they have no competing interests.

\section{References}

1. Siegel R, Naishadham D, Jemal A. Cancer statistics, 2013. CA Cancer J Clin. 2013;63(1):11-30. doi:10.3322/caac.21166

2. Ramalingam SS, Owonikoko TK, Khuri FR. Lung cancer: new biological insights and recent therapeutic advances. CA Cancer J Clin. 2011;61(2):91-112. doi:10.3322/caac.20102

3. Wistuba II. Genetics of preneoplasia: lessons from lung cancer. Curr Mol Med. 2007;7(1):3-14. doi:10.2174/156652407779940468

4. Verdecchia A, Francisci S, Brenner H, et al. Recent cancer survival in Europe: a 2000-02 period analysis of EUROCARE-4 data. Lancet Oncol. 2007;8(9):784-796. doi:10.1016/S1470-2045(07)70246-2

5. Schrump DS, Nguyen DM. Targets for molecular intervention in multistep pulmonary carcinogenesis. World J Surg. 2001;25 (2):174-183. doi: $10.1007 / \mathrm{s} 002680020016$ 
6. Li X, Cao Y, Gong X, Li H. Long noncoding RNAs in head and neck cancer. Oncotarget. 2017;8(6):10726-10740. doi:10.18632/ oncotarget. 12960

7. McAninch D, Roberts CT, Bianco-Miotto T. Mechanistic insight into long noncoding RNAs and the placenta. Int J Mol Sci. 2017;18 (7):1371. doi:10.3390/ijms18071371

8. Soci UPR, Melo SFS, Gomes JLP, Silveira AC, Nobrega C, de Oliveira EM. Exercise training and epigenetic regulation: multilevel modification and regulation of gene expression. Adv Exp Med Biol. 2017;1000:281-322. doi:10.1007/978-981-10-4304-8_16

9. Cusanelli E, Chartrand P. Telomeric repeat-containing RNA TERRA: a noncoding RNA connecting telomere biology to genome integrity. Front Genet. 2015;6:143. doi:10.3389/fgene.2015.00143

10. Benetatos L, Voulgaris E, Vartholomatos G. The crosstalk between long non-coding RNAs and PI3K in cancer. Med Oncol. 2017;34 (3):39. doi:10.1007/s12032-017-0897-2

11. Huang T, Wang X, Yang X, et al. Long non-coding RNA DUXAP8 enhances renal cell carcinoma progression via downregulating miR-126. Med Sci Monit. 2018;24:7340-7347. doi:10.12659/ MSM.910054

12. Jiang B, Hailong S, Yuan J, et al. Identification of oncogenic long noncoding RNA SNHG12 and DUXAP8 in human bladder cancer through a comprehensive profiling analysis. Biomed Pharmacother. 2018;108:500-507. doi:10.1016/j.biopha.2018.09.025

13. Jiang $H$, Shi X, Ye G, et al. Up-regulated long non-coding RNA DUXAP8 promotes cell growth through repressing Kruppel-like factor 2 expression in human hepatocellular carcinoma. Onco Targets Ther. 2019;12:7429-7436. doi:10.2147/OTT.S214336

14. Shivdasani RA. MicroRNAs: regulators of gene expression and cell differentiation. Blood. 2006;108(12):3646-3653. doi:10.1182/blood2006-01-030015

15. Pillai RS, Bhattacharyya SN, Filipowicz W. Repression of protein synthesis by miRNAs: how many mechanisms? Trends Cell Biol. 2007;17(3):118-126. doi:10.1016/j.tcb.2006.12.007

16. Donadeu FX, Schauer SN, Sontakke SD. Involvement of miRNAs in ovarian follicular and luteal development. J Endocrinol. 2012;215 (3):323-334. doi:10.1530/JOE-12-0252

17. Rutnam ZJ, Yang BB. The involvement of microRNAs in malignant transformation. Histol Histopathol. 2012;27(10):1263-1270. doi:10.14670/HH-27.1263

18. Farazi TA, Hoell JI, Morozov P, Tuschl T. MicroRNAs in human cancer. Adv Exp Med Biol. 2013;774:1-20. doi:10.1007/978-94-0075590-1_1

19. Tan S, Shi H, Ba M, et al. miR-409-3p sensitizes colon cancer cells to oxaliplatin by inhibiting Beclin-1-mediated autophagy. Int $J$ Mol Med. 2016;37(4):1030-1038. doi:10.3892/ijmm.2016.2492

20. Zhang G, Liu Z, Xu H, Yang Q. miR-409-3p suppresses breast cancer cell growth and invasion by targeting Akt1. Biochem Biophys Res Commun. 2016;469(2):189-195. doi:10.1016/j.bbrc.2015.11.099

21. Qu R, Chen X, Zhang C. LncRNA ZEB1-AS1/miR-409-3p/ZEB1 feedback loop is involved in the progression of non-small cell lung cancer. Biochem Biophys Res Commun. 2018;507(1-4):450-456. doi:10.1016/j.bbrc.2018.11.059

22. Hanahan D, Weinberg RA. Hallmarks of cancer: the next generation. Cell. 2011;144(5):646-674. doi:10.1016/j.cell.2011.02.013

23. Vander Heiden MG, Cantley LC, Thompson CB. Understanding the Warburg effect: the metabolic requirements of cell proliferation. Science. 2009;324(5930):1029-1033. doi:10.1126/science.1160809

24. Mirebeau-Prunier D, Le Pennec S, Jacques C, et al. Estrogen-related receptor alpha modulates lactate dehydrogenase activity in thyroid tumors. PLoS One. 2013;8(3):e58683. doi:10.1371/journal. pone. 0058683
25. Jiao L, Zhang HL, Li DD, et al. Regulation of glycolytic metabolism by autophagy in liver cancer involves selective autophagic degradation of HK2 (hexokinase 2). Autophagy. 2018;14(4):671-684. doi: $10.1080 / 15548627.2017 .1381804$

26. Xi F, Ye J. Inhibition of lung carcinoma A549 cell growth by knockdown of Hexokinase 2 in situ and in vivo. Oncol Res. 2016;23(1-2):53-59. doi:10.3727/096504015X14459480491740

27. Liu X, Yang Z, Chen Z, et al. Effects of the suppression of lactate dehydrogenase $\mathrm{A}$ on the growth and invasion of human gastric cancer cells. Oncol Rep. 2015;33(1):157-162. doi:10.3892/or.2014.3600

28. Chen Y, Li C, Pan Y, et al. The emerging role and promise of long noncoding RNAs in lung cancer treatment. Cell Physiol Biochem. 2016;38(6):2194-2206. doi:10.1159/000445575

29. Zhao X, Hao S, Wang M, Xing D, Wang C. Knockdown of pseudogene DUXAP8 expression in glioma suppresses tumor cell proliferation. Oncol Lett. 2019;17(3):3511-3516. doi:10.3892/ ol.2019.9994

30. Sun M, Nie FQ, Zang C, et al. The pseudogene DUXAP8 promotes non-small-cell lung cancer cell proliferation and invasion by epigenetically silencing EGR1 and RHOB. Mol Ther. 2017;25(3):739-751. doi:10.1016/j.ymthe.2016.12.018

31. Agathocleous M, Harris WA. Metabolism in physiological cell proliferation and differentiation. Trends Cell Biol. 2013;23(10):484-492. doi:10.1016/j.tcb.2013.05.004

32. Hua Q, Mi B, Huang G. The emerging co-regulatory role of long noncoding RNAs in epithelial-mesenchymal transition and the Warburg effect in aggressive tumors. Crit Rev Oncol Hematol. 2018;126:112-120. doi:10.1016/j.critrevonc.2018.03.028

33. Hung CL, Wang LY, Yu YL, et al. A long noncoding RNA connects c-Myc to tumor metabolism. Proc Natl Acad Sci U S A. 2014;111 (52):18697-18702. doi:10.1073/pnas.1415669112

34. Hua Q, Jin M, Mi B, et al. LINC01123, a c-Myc-activated long non-coding RNA, promotes proliferation and aerobic glycolysis of non-small cell lung cancer through miR-199a-5p/c-Myc axis. J Hematol Oncol. 2019;12(1):91. doi:10.1186/s13045-019-0773-y

35. Li Z, Li X, Wu S, Xue M, Chen W. Long non-coding RNA UCA1 promotes glycolysis by upregulating hexokinase 2 through the mTOR-STAT3/microRNA143 pathway. Cancer Sci. 2014;105 (8):951-955. doi:10.1111/cas.12461

36. Ooi AT, Gomperts BN. Molecular pathways: targeting cellular energy metabolism in cancer via inhibition of SLC2A1 and LDHA. Clin Cancer Res. 2015;21(11):2440-2444. doi:10.1158/1078-0432.CCR14-1209

37. Mukherjee A, Ma Y, Yuan F, et al. Lysophosphatidic acid up-regulates Hexokinase II and glycolysis to promote proliferation of ovarian cancer cells. Neoplasia. 2015;17(9):723-734. doi:10.1016/ j.neo.2015.09.003

38. Li LQ, Yang Y, Chen H, Zhang L, Pan D, Xie WJ. MicroRNA-181b inhibits glycolysis in gastric cancer cells via targeting hexokinase 2 gene. Cancer Biomark. 2016;17(1):75-81. doi:10.3233/CBM-160619

39. Zhu W, Huang Y, Pan Q, Xiang P, Xie N, Yu H. MicroRNA-98 suppress Warburg effect by targeting HK2 in colon cancer cells. Dig Dis Sci. 2017;62(3):660-668. doi:10.1007/s10620-016-4418-5

40. Li L, Liu H, Du L, et al. miR-449a suppresses LDHA-mediated glycolysis to enhance the sensitivity of non-small cell lung cancer cells to ionizing radiation. Oncol Res. 2018;26(4):547-556. doi:10.3727/096504017X15016337254605

41. Song Q, Ji Q, Xiao J, et al. miR-409 inhibits human non-small-cell lung cancer progression by directly targeting SPIN1. Mol Ther Nucleic Acids. 2018;13:154-163. doi:10.1016/j.omtn.2018.08.020

42. Wan L, Zhu L, Xu J, et al. MicroRNA-409-3p functions as a tumor suppressor in human lung adenocarcinoma by targeting c-Met. Cell Physiol Biochem. 2014;34(4):1273-1290. doi:10.1159/000366337 


\section{Publish your work in this journal}

OncoTargets and Therapy is an international, peer-reviewed, open access journal focusing on the pathological basis of all cancers, potential targets for therapy and treatment protocols employed to improve the management of cancer patients. The journal also focuses on the impact of management programs and new therapeutic agents and protocols on patient perspectives such as quality of life, adherence and satisfaction. The manuscript management system is completely online and includes a very quick and fair peer-review system, which is all easy to use. Visit http://www.dovepress.com/ testimonials.php to read real quotes from published authors. 